\title{
A Survey on Methods for Computing Matrix Exponentials in Numerical Schemes for ODEs
}

\author{
Nicoletta Del Buono and Luciano Lopez \\ Dipartimento Interuniversitario di Matematica, \\ Università degli Studi di Bari \\ Via E. Orabona, 4 - I-70125 Bari ITALY \\ \{delbuono, lopezl\}@dm. uniba.it
}

\begin{abstract}
This paper takes a look at numerical procedures for computing approximation of the exponential of a matrix of large dimension. Existing approximation methods to evaluate the exponentiation of a matrix will be reviewed paying more attention to Krylov subspace methods and Schur factorization techniques. Some theoretical results on the bounds for the entries of the exponential matrix and some implementation details will be also discussed.
\end{abstract}

\section{Introduction}

Several problems in mathematics and physics can be formulated in terms of finding a suitable approximations to certain matrix functions. Particularly, the issue of computing the matrix exponential $f(A)=e^{-t A}, t \geq t_{0}$, is one of the most frequently encountered tasks in matrix function approximation and it has received a renew attention from the numerical analysis community. This problem arises in many areas of applications, as for instance the solution of linear parabolic partial differential equations which needs the numerical solution of $n$ dimensional systems of ODEs $\dot{y}=-A y+b(t), y(0)=y_{0}, t>0$ [31516, or recently in the field of geometric integration. In fact, most Lie group methods, as Runge-Kutta/Munthe-Kaas schemes, Magnus expansions and Fer expansions [192028], require to suitable approximate the matrix exponential from a Lie algebra $g \subset \mathbb{R}^{n \times n}$ once (and often repeatedly) at each time step. This can be a very challenging task for large dimension matrix. Moreover, the context of Liegroup method imposes a crucial extra requirement on the approximant: it has to reside in the Lie group $G \subset G L(n, \mathbb{R})$ associated to the Lie algebra $g$.

In general, this property is not fulfilled by many standard approximations unless the exponential is evaluated exactly.

This can be done for instance for a $3 \times 3$ skew symmetric matrices, whose exponential is given exactly by the Euler Rodriguez formula

$$
\exp (A)=I+\frac{\sin (\alpha)}{\alpha} A+\frac{1}{2}\left(\frac{\sin (\alpha / 2)}{\alpha / 2}\right)^{2} A^{2},
$$

where 


$$
A=\left(\begin{array}{ccc}
0 & a_{3} & -a_{2} \\
-a_{3} & 0 & a_{1} \\
a_{2} & -a_{1} & 0
\end{array}\right), \quad \alpha=\left(a_{1}^{2}+a_{2}^{2}+a_{3}^{2}\right)^{1 / 2} .
$$

Other exact formulas for exponentials of skew symmetric matrices can be obtained making use of the Cayley-Hamilton theorem which for every $A \in$ $G L(n, \mathbb{R})$ allows us to have the basic decomposition $\exp (t A)=\sum_{k=0}^{m-1} f_{k}(t) A^{k}$, where $m$ is the degree of the minimal polynomial of $A$ and $f_{0}, \ldots, f_{m-1}$ are some analytic functions that depend on the characteristic polynomial of $A$. However, these algorithms are practical only up to dimension eight. In fact, for large scale matrices, they require the computation of high powers of the matrix in question and hence high computational costs due to the matrix-matrix multiplication; moreover they suffer of some computational instabilities due to the direct use of the characteristic polynomial.

Based on the above remarks, it appears clear that exponential of large matrices cannot be evaluated analytically and that an algorithm which is simple and efficient and satisfies some geometric properties is highly desiderable.

In the following pages, we will review some existing approximation methods, highlighting also some theoretical aspects. In the last section we will give also a brief overview on a new methodology with can be consider an hybrid scheme for exponential approximation.

\section{Review of Existing Approximation Methods}

Classical methods for the evaluation of a matrix exponential can be classified into three main categories:

(i) rational approximants;

(ii) Krylov subspace methods;

(iii) techniques based on numerical linear algebra;

further, within the context of Lie-group theory other methods for the approximation of the exponential have been recently introduced:

(iv) the splitting methods.

\subsection{Rational Approximants}

A rational approximation of the exponential function replaces it by a rational function, $\exp (z) \approx r(z):=p_{\alpha}(z) / q_{\beta}(z)$, where $p$ and $q$ are polynomials of degree $\alpha$ and $\beta$ respectively, $q(0)=1$ and the error term $e^{z}-p(z) / q(z)$ is small in a chosen norm. Thus for the matrix $\exp (A)$ one has to compute two matrix-value polynomials, $p(A)$ and $q(A)$, and invert the latter to obtain $r(A)$.

Probably, the most popular approximant of this kind is the diagonal $(\nu, \nu)$ Padé approximations where

$$
p_{\nu}(z)=\sum_{k=1}^{\nu} \frac{(2 \nu-k) ! \nu !}{2 \nu ! k !(\nu-k) !} z^{k} \quad \text { and } \quad q_{\nu}(z)=p_{\nu}(-z) .
$$


Unfortunately, the Padé approximants are good only near the origin (see [13]). However, this problem can be overcome with the so-called scaling and squaring technique, which exploits the identity

$$
\exp (A)=\left(\exp \left(A / 2^{k}\right)\right)^{2^{k}}
$$

as follows. First, a sufficiently large $k$ is chosen so that $A / 2^{k}$ is close to 0 , then a diagonal Padé approximant is used to calculate $\exp \left(A / 2^{k}\right)$, and finally the result is squared $k$ times to obtain the required approximation to $\exp (A)$. This basic approach is implemented in the Matlab function expm to evaluate the exponential of a matrix with a computational cost between $20 n^{3}$ and $30 n^{3}$ operations.

About the behavior of rational approximants when applied to matrix belonging to a Lie algebra, the following theorem states that an important category of Lie group leads itself to suitable rational approximations.

Theorem 1. (see [5]) Let $G=\left\{Y \in G L(n, \mathbb{R}): Y^{T} P Y=P\right\}$, where $P$ is a non-singular $n \times n$ matrix and let $g=\left\{X \in g l(n, \mathbb{R}): X P+P X^{T}=0\right\}$ be the corresponding Lie algebra. Let $\phi$ be a function analytic in a neighborhood $U_{0}$ of 0 , with $\phi(0)=1$ and $\phi^{\prime}(0)=1$. If

$$
\phi(z) \phi(-z)=1, \quad \forall z \in U_{0},
$$

then $\forall X \in g, \Phi(t X) \in G$ for all $t \in \mathbb{R}^{+}$sufficiently small.

Examples of Lie group verifying the above theorem are the orthogonal and the symplectic group. Moreover, the diagonal Padé approximants are analytic functions that fulfill (11), and this guarantees that their approximation of the exponential of a matrix in the Lie-algebra of a quadratic Lie group lies in the corresponding group.

Another class of approximations of $\exp (A)$ are based on the Chebyshev series expansion of the function $\exp (z)$ on the spectral interval of $A$. Now let $A$ be an Hermitian matrix and suppose that $e^{z}$ is analytic in a neighborhood of the spectral interval $\left[\lambda_{1}, \lambda_{n}\right]$ of $A$. The approximation of $\exp (A) v$ has the form

$$
\exp (A) v \approx \sum_{k=0}^{m} a_{k} C_{k}\left(\frac{1}{l_{1}} A-\frac{1}{l_{2}} I\right) v,
$$

where $l_{1}=\left(\lambda_{n}-\lambda_{1}\right) / 2$ and $l_{2}=\left(\lambda_{n}+\lambda_{1}\right) / 2, C_{k}$ is the $k$ th Chebyshev polynomial of the first kind and the $a_{k}$ are the Chebyshev coefficients of $e^{\left(l_{1} t+l_{2}\right)}$, $t \in[-1,1]$. Chebyshev polynomial approximation for the exponential of symmetric large sparse matrices was considered in [34, while in 2] a lower bound for $\|\exp (-\tau A) v\|_{2}$ based on the computation of an eigenvector associated with $\lambda_{1}$ was obtained. The exponential of a non-symmetric matrix has also been approximated in [26|27] using the Faber polynomial and the Faber series. 


\subsection{Methods of Numerical Linear Algebra}

A simple technique to evaluate $\exp (A)$ is by spectral decomposition: if $A=$ $V D V^{-1}$ where $D$ is diagonal matrix with diagonal elements $\lambda_{i}, i=1, \ldots, n$, then

$$
\exp (A)=V \exp (D) V^{-1}=V\left(\begin{array}{cccc}
e^{\lambda_{1}} & 0 & \ldots & 0 \\
0 & e^{\lambda_{2}} & & \\
\vdots & \ddots & & \\
& & & \ldots e^{\lambda_{n}}
\end{array}\right) V^{-1}
$$

This, however, is not a viable techniques for general matrices. In place of spectral decomposition, one can factorize $A$ in a different form, e.g. into Schur decomposition (see 13]) $A=Q T Q^{T}$, where $Q$ is an orthogonal $n \times n$ matrix and $T$ is an $n \times n$ upper triangular matrix or a block upper triangular matrix (when the eigenvalue of $A$ can be clustered in blocks) and therefore $\exp (A)=Q \exp (T) Q^{T}$.

\subsection{Krylov Subspace Methods}

The basic idea of the Krylov subspace techniques is to project the exponential of the large matrix onto a small Krylov subspace. Particularly, an approximation to the matrix exponential operation $\exp (A) v$ of the form

$$
\exp (A) v \approx p_{m-1}(A) v,
$$

where $A \in G L(n, \mathbb{R}), v$ is an arbitrary nonzero vector, and $p_{m-1}$ is a polynomial of degree $m-1$. Since this approximation is an element of the Krylov subspace

$$
K_{m}(A, v)=\operatorname{span}\left\{v, A v, \cdots, A^{m-1} v\right\},
$$

the problem can be reformulated as that of finding an element of $K_{m}(A, v)$. To find such an approximation can be used the Arnoldi and nonsymmetric Lanczos algorithms, respectively. The Arnoldi algorithm generates an orthogonal basis of the Krylov subspace using $v_{1}=v /\|v\|$ as initial vector.

\section{Arnoldi algorithm}

1. Compute $v_{1}=v /\|v\|$.

2. Do $j=1,2, \ldots, m$.

$w:=A v_{j}$

Do $i=1,2, \ldots, j$

$$
h_{i, j}:=\left(w, v_{j}\right) \quad w:=w-h_{i, j} v_{j}
$$

Compute $h_{j+1}=\|w\|_{2}$ and $v_{j+1}=w / h_{j+1, j}$.

Arnoldi algorithm produces an orthonormal basis $V_{m}=\left[v_{1}, v_{2}, \ldots, v_{m}\right]$ of the Krylov subspace $K_{m}(A, v)$ and a $m \times m$ upper Hessemberg matrix $H_{m}$ with elements $h_{i, j}$ such that

$$
A V_{m}=V_{m} H_{m}+h_{m+1, m} v_{m+1} e_{m}^{T}
$$


from which we get $H_{m}=V_{m}^{T} A V_{m}$. Therefore $H_{m}$ represents the projection of the linear transformation $A$ onto the subspace $K_{m}$, with respect to the basis $V_{m}$. Based on this we can also write $\exp (A) v \approx \beta V_{m} \exp \left(H_{m}\right) e_{1}$ (where $\beta=$ $\left.\|v\|_{2}\right)$. Now, the problem of computing $\exp (A) v$ has been reduced to the task of computing the lower-dimensional expression $\exp \left(H_{m}\right) e_{1}$, which for $m \ll n$ is usually much easier, e.g., by diagonalization of $H_{m}$.

The computational cost required by the Krylov subspace methods with the Arnoldi iteration is sum of the following partial amounts of operations, counting both multiplications and additions: $2 m n^{2}+2 n m(m-1)$ operation to compute the Krylov subspace $K_{m}(A, v)$ of dimension $m ; \mathrm{Cm}^{3}$ computations for the evaluation of the exponential of the Hessemberg matrix $H_{m} ; 2 n m$ operations arising from the multiplication of $\exp \left(H_{m}\right)$ with the orthogonal basis. However, when $n$ is large and $m \ll n$, these costs are subsumed in that of the Arnoldi iteration, and the leading factor is $2 m n^{2}$ ( $2 m n^{3}$ for matrices).

Some error bounds for the Arnoldi approximation of $\exp (A) v$ can be given for various classes of matrices.

Theorem 2. (see [17]) Let $A$ be a complex square matrix of large dimension $n$, and $v$ a given $n$-dimensional vector of unit length $(\|v\|=1)$ and $e r r_{m}=\left\|\exp (\tau A) v-V_{m} \exp \left(\tau H_{m}\right) e_{1}\right\|$ be the error in the Arnoldi approximation of $\exp (\tau A)$. Then err $_{m}$ satisfies the following bounds:

(1) if $A$ is Hermitian negative semidefinite matrix with eigenvalues in the interval $[-4 \rho, 0]$ then:

$$
\begin{array}{ll}
\operatorname{err}_{m} \leq 10 e^{-m^{2} / 5 \rho \tau}, & \sqrt{4 \rho \tau} \leq m \leq 2 \rho \tau, \\
\operatorname{err}_{m} \leq 10(\rho \tau)^{-1} e^{-\rho \tau}(e \rho \tau / m)^{m}, & m \geq 2 \rho \tau
\end{array}
$$

(2) if $A$ is skew-Hermitian matrix with eigenvalues in an interval on the imaginary axis of length $4 \rho$, then

$$
e r r_{m} \leq 12 e^{-(\rho \tau)^{2} / m}(e \rho \tau / m)^{m}, \quad m \geq 2 \rho \tau ;
$$

(3) if $A$ has numerical range contained in the disk $|z+\rho| \leq<\rho$, then:

$$
e r r_{m} \leq 12 e^{-\rho \tau}(e \rho \tau / m)^{m}, \quad m \geq 2 \rho \tau
$$

Another well known algorithm for constructing a convenient basis of $K_{m}$ is the Lanczos algorithm which, starting from two vectors $v_{1}$ and $w_{1}$, generates a biorthogonal basis of the subspaces $K_{m}\left(A, v_{1}\right)$ and $K_{m}\left(A^{T}, w_{1}\right)$.

\section{Lanczos algorithm}

1. Compute $v_{1}=v /\|v\|$ and select $w_{1}$ so that $\left(v_{1}, w_{1}\right)=1$.

2. Do $j=1,2, \ldots, m$.

$$
\begin{aligned}
& \alpha_{j}:=\left(A v_{j}, w_{j}\right) \\
& \hat{v}_{j+1}:=A v_{j}-\alpha_{j} v_{j}-\beta_{j} v_{j-1} \\
& \hat{w}_{j+1}:=A^{T} w_{j}-\alpha_{j} w_{j}-\delta_{j} w_{j-1} \\
& \beta_{j+1}:=\sqrt{\left|\left(\hat{v}_{j+1}, \hat{w}_{j+1}\right)\right|}, \delta_{j+1}:=\beta_{j+1} \operatorname{sign}\left[\left(\hat{v}_{j+1}, \hat{w}_{j+1}\right)\right] \\
& v_{j+1}=\hat{v}_{j+1} / \delta_{j+1} \text { and } w_{j+1}=\hat{w}_{j+1} / \beta_{j+1} .
\end{aligned}
$$


Setting $V_{m}=\left[v_{1}, v_{2}, \ldots, v_{m}\right], W_{m}=\left[w_{1}, w_{2}, \ldots, w_{m}\right]$ and $T_{m}=$ $\operatorname{tridiag}\left(\delta_{i}, \alpha_{i}, \beta_{i}\right)$ then $W_{m}^{T} V_{m}=V_{m} W_{m}=I$, with $I$ the identity matrix and

$$
A V_{m}=V_{m} T_{m}+\delta_{m+1} v_{m+1} e_{m}^{T}
$$

from which we can derive the approximation $\exp (A) v \approx \beta V_{m} \exp \left(T_{m}\right) e_{1}$. The exponential matrix $\exp \left(T_{m}\right)$ can be computed again from the eigendecomposition $T_{m}=Q_{m} D_{m} Q_{m}^{T}$, with diagonal $D_{m}$, via $\exp \left(T_{m}\right)=Q_{m} \exp \left(D_{m}\right) Q^{T}$.

Observe, that both these methods reduce to the same technique when the matrix is symmetric.

For the Lanczos method it can also be proved analogous error bounds (except for different constants) as that given for the Arnoldi method in Theorem 2 (see [17]).

\subsection{Splitting Methods}

Splitting methods have been considered by various authors in different contexts: for constructing symplectic methods or volume preserving algorithms, and in PDE context 5715]. A good survey can be found in [24].

The idea these methods are based on is the following: a given $n \times n$ matrix $A$ in a Lie algebra $g$ is split in the form

$$
A=\sum_{k=1}^{s} A_{k},
$$

where $A_{k} \in g, k=1,2, \ldots, s$, has an exponential that can be easily evaluated exactly so that

$$
\exp \left(t A_{1}\right) \exp \left(t A_{2}\right) \cdots \exp \left(t A_{s}\right)=\exp (t A)+O\left(t^{p+1}\right)
$$

for sufficiently large value of $p \geq 1$. Of course this procedure is competitive with direct evaluations of $\exp (t A)$ only when each $\exp \left(t A_{i}\right)$ is easy to evaluate exactly and their products are cheap.

These requirements are satisfies when each $C=A_{i}$ is a low rank matrix, i.e. $C=\sum_{l=1}^{p} \alpha_{l} \beta_{l}^{T}=\alpha \beta^{T}$, with $\alpha_{l}, \beta_{l} \in \mathbb{R}^{n}$, where $p \geq 1$ is a small integer and $\alpha=\left[\alpha_{1}, \ldots, \alpha_{p}\right], \beta=\left[\beta_{1}, \ldots, \beta_{p}\right]$ are $n \times p$ matrices. In this case, the function $\exp (t C)$ can be calculated explicitely via the formula

$$
\exp (t C)=I+\alpha D^{-1}(\exp (t D)-I) \beta^{T} .
$$

where $D=\beta^{T} \alpha$ is nonsingular (see [5]). Observe that each $\exp (t D)$ can be approximated with a diagonal $(\nu, \nu)$-Padé approximant at the cost of $\nu p^{3}$ flops.

This low-rank splitting can be easy generalized splitting $A$ into matrices having one raw and one column (or a few rows and a few columns) (see 5] for more details). Recently, it has been demonstrated also that the splitting (2) can be improved when $A$ belongs to a Lie algebra $g$ by letting

$$
A=\sum_{k=1}^{r} a_{k} Q_{k}
$$


where $r=\operatorname{dim}(g)$ and $Q_{1}, Q_{2}, \ldots, Q_{r}$ is a basis of the algebra, and

$$
\exp (t A) \approx \exp \left(g_{1}(t) Q_{1}\right) \exp \left(g_{2}(t) Q_{2}\right) \ldots \exp \left(g_{r}(t) Q_{r}\right)
$$

and $g_{1}, g_{2}, \ldots, g_{r}$ are polynomials $([6])$. In this case, the right choice of the basis and the use of certain features of the Lie algebra assume a fundamental importance [6].

\section{A Recent Approach Based on SVD Techniques}

Recently, other approaches for evaluating both $\exp (A)$ and $\exp (\tau A) y$ where $A$ is a sparse skew symmetric matrix of large dimension $n, y$ is a given vector, and $\tau$ is a scaling factor, have been proposed which takes advantage from some of the approaches discussed before. In [9] a procedure based on an effective Schur decomposition of the skew symmetric matrix $A$ consisting into two main steps is presented. We will describe briefly this method. In the first step the skewsymmetric matrix $A$ is tranformed via the Arnoldi procedure into its Hessenberg form $H$, e.g. $A=Q H Q^{T}$ with $Q n \times n$ orthogonal matrix and $H$ an Hessenberg $n \times n$ matrix possessing a tridiagonal structure. Then in the second step a Schur decomposition of $H$ is obtained by using the singular value decomposition of a bidiagonal matrix of half size of $H$. The proposed procedure allows to take full advantage of the sparsity of $A$ and of the tridiagonal form of $H$. In fact, the main cost for evaluating $\exp (A)$ is $\frac{9}{2} n^{3}$ flops which reduces to $2 n^{3}$ flops when the banded structure of $H$ is exploited; further, $k n^{2}$ flops are needed for evaluating $\exp (\tau A) y$.

Remark 1. In the implementation of a method to approximate the exponential of a matrix it could be important to know whether the entries of the matrix function $\exp (A)$ exhibit some kind of decay behavior away from the main diagonal, and to be able to estimate the rate of decay. Generally, even $A$ is a sparse, $\exp (A)$ is usually a dense matrix. However when the matrix in study presents a banded structure it can be proved that the elements of the exponential decay very rapidly away from the diagonal and in practical computation they can be set to zero away from some bandwidth which depends on the required accuracy threshold (see [1 21]. Let $A$ be a banded matrix of bandwidth $s \geq 1$ then the extra diagonal entries of $\exp (A)$ rapidly decay, as

$$
\left|[\exp (A)]_{k, l}\right|<\exp (\rho) I_{|k-l|}(2 \rho), \quad \text { for } k, l=1,2, \ldots, n,
$$

where $[\exp (A)]_{k, l}$ denotes the $(k, l)$ entry of the matrix $\exp (A), \rho=\max _{2 \leq j \leq n} h_{j}$ and $I_{r}(z)$ is the modified Bessel function with $I_{r}(2 \rho) \cong \frac{\rho^{r}}{r !}$ for $r>>1$ (see [21] for details). It not always clear at all how, for a bounded $A$, the nearness of $\exp (A)$ to a banded matrix can be exploited for improving Krylov subspace approximation or into rational approximation, but it has been used successfully in the context of splitting methods and in linear algebra methods to save some computational costs. 


\section{Exponentiation in Numerical Methods for ODEs}

As observed above, the use of exponential of matrices for the numerical integration of ordinary differential equations on manifolds has been successfully adopted for designing a large number of geometrical methods which behave more favorably than standard integrators (see for instance 14]). In this final section, we will report some geometric integrators for linear and nonlinear differential equations.

Second and fourth order Magnus methods for $y^{\prime}=A(t) y$

$$
\begin{array}{ll}
\text { MG2 } & \text { MG4 } \\
A_{1}=A\left(t_{n}+h / 2\right) ; & A_{1}=A\left(t_{n}+\left(\frac{1}{2}-\frac{\sqrt{3}}{6}\right) h\right) \\
\omega_{n}=A_{1} ; & A_{2}=A\left(t_{n}+\left(\frac{1}{2}+\frac{\sqrt{3}}{6}\right) h\right) \\
y_{n+1}=\exp \left(h \omega_{n}\right) y_{n} & \omega_{n}=\frac{1}{2}\left(A_{1}+A_{2}\right)+\frac{\sqrt{3}}{12} h\left[A_{2}, A_{1}\right] ; \\
& y_{n+1}=\exp \left(h \omega_{n}\right) y_{n}
\end{array}
$$

Third order Crouch-Grossman method for $y^{\prime}=A(t, y) y$ :

$$
\begin{aligned}
& A_{1}=A\left(t_{n}, y_{n}\right) \\
& A_{2}=A\left(t_{n}+\frac{3}{4} h, \exp \left(\frac{3}{4} h A_{1}\right) y_{n}\right) ; \\
& A_{3}=A\left(t_{n}+\frac{3}{4} h, \exp \left(\frac{1}{108} h A_{2}\right) \exp \left(\frac{119}{216} h A_{1}\right) y_{n}\right) ; \\
& y_{n+1}=\exp \left(\frac{13}{51} h A_{3}\right) \exp \left(-\frac{2}{3} h A_{2}\right) \exp \left(\frac{24}{17} h A_{1}\right) y_{n}
\end{aligned}
$$

Exponential methods for nonlinear initial value problem $y^{\prime}=f(y)$

- The exponential fitted Euler method:

$$
y_{n+1}=y_{n}+h \varphi(h A) f\left(y_{n}\right),
$$

- A symmetric exponential method:

$$
y_{n+1}-y_{n}=\exp (h A)\left(y_{n-1}-y_{n}\right)+2 h \varphi(h A) f\left(y_{n}\right),
$$

where with $A=f^{\prime}\left(y_{n}\right)$ is Jacobian matrix of $f$ and $\varphi=\frac{e^{z}-1}{z}$.

\section{References}

1. Benzi M., Golub G.H.: Bounds fro the entries of matrix functions with applications to preconditioning, BIT 39 (3) (1999) pp 417-438.

2. Bergamaschi, L., Vianello, M.: Efficient computation of the exponential operator for large, sparse, symmetric matrices. Numer. Linear Algebra Appl. 7 (2000), pp. $27-45$.

3. Calvetti D., Reichel L.: Exponential Integration methods for large stiff systems of differential equations. Iterative Methods in Scientific Computation II (1999), pp $1-7$. 
4. Castillo P., Saad Y.: Preconditioning the matrix exponential operator with applications. J. Sci. Comput. 13(3) (1998), pp. 275-302.

5. Celledoni E., Iserles A.: Approximating the exponential from a Lie algebra to a Lie group. Math. Comp. 69 (2000), pp. 1457-1480.

6. Celledoni E., Iserles A.: Numerical calculation of the exponential based on WeiNorman equation Tech. Report, University of Cambridge.

7. Celledoni E., Iserles A.: Methods for the approximation of the matrix exponential in a Lie-algebraic setting. IMA J. Numer. Anal., No. 21, (2001) pp. 463-488.

8. Cheng, H.-W., Yau S.S.T.: More explicit formulas for the matrix exponential. Linear Algebra Appl. 262 (1997), pp. 131-163.

9. Del Buono N., Lopez L., Peluso R.: Computation of Exponentials of Real Skew Symmetric Matrices by SVD Techniques, Tech. Report 7/03 (2003).

10. Dieci L., Papini A.: Padé approximation for the exponential of a block triangular matrix. Linear Algebra Appl. 308 (2000), pp. 183-202.

11. Dieci L., Papini A.: Conditioning of the exponential of a block triangular matrix. Numer. Algorithms 28, (2001), pp. 137-150.

12. Gallopoulos E., Saad Y.: Efficient solution of parabolic equations by Krylov approximation methods, SIAM J. Sci. Stat. Comput. 13, No. 5, pp. 1236-1264, 1992.

13. Golub G.H., van Loan C.F.: Matrix Computation. 3th edn, John Hopikns, Baltimore.

14. Hairer E., Lubich C., Wanner. G.: Geometric Numerical Integration: StructurePreserving Algorithms for Ordinary Differential Equations. Springer-Verlag, Berlin, 2002.

15. Hochbruck M., Lubich C.: Exponential integrators for quantum-classical molecular dynamics, BIT 39, No.4, pp. 620-645, 1999.

16. Hochbruck M., Lubich C.: Exponential integrators for large systems of differential equations, SIAM J. Sci. Comput. 19, No.5, pp. 1552-1574, 1998.

17. Hochbruck M., Lubich C.: On Krylov subspace approximations to the matrix exponential operator SIAM J. Numer. Anal. 34, No.5, pp. 1911-1925, (1997).

18. Horn R., Johnson C.: Topics in Matrix Analysis. Cambridge University Press, New York, 1995.

19. Iserles, A., Nørsett S.P., Rasmussen A.F.: Time symmetry and high-order Magnus methods. Appl. Numer. Math. 39, (2001) 379-401.

20. Iserles A., Munthe-Kaas H., Nørsett S., Zanna A.: Lie-group methods, Acta numerica. Cambridge: Cambridge University Press. Acta Numer. 9, 215-365 (2000).

21. Iserles A.: How large is the exponential of a banded matrix? N. Z. J. Math. 29, No.2, pp. 177-192 (2000).

22. Iserles A., Zanna A.: Efficient computation of the matrix exponential by Generalized Polar Decompositions. DAMTP Technical Report NA2002/09, University of Cambridge, UK (2002).

23. Leite F.S., Crouch, P.: Closed forms for the exponential mapping on matrix Lie groups based on Putzer's method. J. Math. Phys. 40, No.7, pp. 3561-3568, 1999.

24. McLachlan, R. I., Quispel G.R.W.: Splitting methods. Acta Numerica 2002.

25. Moler C.B., Van Loan C.F.: Nineteen dubious ways to compute matrix exponential. SIAM Review 20 (1978), pp. 801-836.

26. Moret I., Novati P.: An interpolatory approximation of the matrix exponential based on Faber polynomials. J. Comput. Appl. Math. 131 (2001) pp. 361-380.

27. Moret I., Novati P.: The computation of functions of matrices by truncated Faber series. Numer. Funct. Anal. Optimization 22, (2001), pp. 697-719.

28. Munthe-Kaas, H.: High order Runge Kutta methods on manifolds. Appl. Numer. Math. 29(1) (1999), pp. 115-127. 
29. Saad Y.: Analysis of some Krylov subspace approximation to the matrix exponential operator, SIAM J. Numer. Anal. 29, No. 1, pp. 209-228, 1992.

30. Van Loan C.: The sensitivity of the matrix exponential. SIAM J. Matrix Anal. Appl. 14 (1977), pp. 971-981.

31. Ben Taher R., Rachidi, M.: Some explicit formulas for the polynomial decomposition of the matrix exponential and applications. Linear Algebra Appl. 350, (2002) pp. 171-184.

32. Politi T.: A formula for the exponential of a real skew-symmetric matrix of order 4. BIT 41, No.4, (2001) pp. 842-845.

33. Tal-Ezer H.: Polynomial approximation of functions of matrices and applications. J. Sci. Comput. 4 (1989), pp. 25-60.

34. Tal-Ezer H.: Spectral methods in time for parabolic problems. SIAM J. Numer. Anal. 26 (1989), pp. 1-11.

35. Zanna A., Munthe-Kaas H.Z.: Generalized polar decompositions for the approximation of the matrix exponential. SIAM J. Matrix Anal. Appl. 23(3) (2002), pp. 840-862. 